\title{
Detección de anticuerpos neutralizantes en profesionales de la salud vacunados contra el SARS-CoV-2
}

Arturo Pareja Cruz 1,a; Joel de León Delgado 1,b; Pedro Javier Navarrete Mejía 1,2,c; Julio César Luque Espino 1,d; Jesús Demóstenes Gonzáles Moscoso 3,4,e

\section{RESUMEN}

Objetivo: Determinar la presencia y concentración de los anticuerpos neutralizantes en el suero de individuos que recibieron la vacuna contra el SARS-CoV-2 desarrollada por la empresa Sinopharm.

Materiales y métodos: Investigación descriptiva y transversal. En el estudio se incluyeron 117 profesionales de la salud inoculados con dos dosis de la vacuna Sinopharm, en quienes se había detectado la presencia de anticuerpos neutralizantes contra SARS-CoV-2 en el suero mediante la técnica de inmunoensayo de electroquimioluminiscencia (eCLIA). Las variables consideradas en el estudio fueron la edad, el sexo, la concentración de anticuerpos neutralizantes y el antecedente de haber tenido COVID-19.

Resultados: Se evidenció el incremento significativo de la concentración de anticuerpos neutralizantes en las personas que recibieron la vacuna y tuvieron una infección previa por SARS-CoV-2 $(p<0,001)$ respecto a las que se vacunaron, pero no presentaron infección previa.

Conclusiones: El estudio evidencia que la vacunación refuerza la inmunidad contra el nuevo coronavirus en los pacientes con diagnóstico previo de COVID-19 y sugiere la relevancia de la aplicación de una tercera dosis de esta vacuna.

Palabras clave: Anticuerpos neutralizantes; COVID-19; Inmunogenicidad; Vacunación; Personal de salud (Fuente: DeCS BIREME).

\section{Detection of neutralizing antibodies on anti-SARS-CoV2 vaccinated healthcare professionals}

\section{ABSTRACT}

Objective: To describe the presence and the concentration of neutralizing antibodies at serum of healthcare professionals inoculated with the Sinopharm anti-SARS-CoV2 vaccine.

Materials and methods: Descriptive and transversal research. A total of 117 healthcare professionals inoculated with two doses of the Sinopharm vaccine having quantitative data of neutralizing antibodies at serum (detected by eCLIA) were included. Age, sex, neutralizing antibodies concentration and previous diagnostic of COVID-19 diseases were the variables. Results: A significant increase on neutralizing antibodies concentration were detected on pre- COVID-19 vaccinated persons regarding those vaccinated without previous diagnostic of the viral infection $(p<0,001)$.

Conclusions: the study evidence that Sinopharm vaccine boost immunity against the new coronavirus on previously infected persons and suggest that a third dose of the vaccine could be relevant to boost the immune response.

Keywords: Antibodies, neutralizing; Coronavirus infections; Immunogenicity, vaccine; Vaccination; Health personnel.

\footnotetext{
1 Universidad de San Martín de Porres, Facultad de Medicina, Instituto de Investigación. Lima, Perú.

2 Universidad Continental. Lima, Perú.

3 Hospital Nacional Daniel Alcides Carrión. Callao, Perú.

4 Centro Gamma Medica Servicios Médicos. Lima, Perú.

a Doctor en Medicina.

b Doctor en Ciencias Biológicas.

c Doctor en Salud Pública.

d Maestro en Investigación Clínica.

e Médico Cirujano, especialista en Patología Clínica.
} 


\section{INTRODUCCIÓN}

En diciembre de 2019, en Wuhan (China), se registró un brote de cuadros con síntomas clínicos comunes de neumonía, tras el ingreso masivo de pacientes en los hospitales locales. Poco después, la Organización Mundial de la Salud (OMS) confirmó la aparición de un nuevo coronavirus, Ilamado SARS-CoV-2, como el agente etiológico de estos síntomas y nombró a esta enfermedad como COVID-19. Desde el año pasado, esta pandemia, sin precedente en las últimas décadas, atrae la atención de muchos médicos, científicos y agencias reguladoras de todo el mundo que buscan conocer la patogenia, técnicas de diagnóstico, posibles terapias y vacunas eficaces contra el agente infeccioso ${ }^{(1,2)}$.

Una gran diversidad de estrategias de inmunoterapia se ha desarrollado e implementado para controlar la infección por SARS-CoV-2 y tratar la enfermedad que ocasiona, la COVID-19 (3,4). En poco más de un año, diversas plataformas vacunales para ser incluidas en estrategias masivas de vacunación han sido diseñadas y evaluadas (en preclínica, clínica y escalado productivo) ${ }^{(5,6)}$. Cada país tiene la libertad de masificar la inmunización contra este virus; sin embargo, y de manera general, se ha optado por priorizar a las personas más expuestas o con alto riesgo de desarrollar una enfermedad grave, es decir, los profesionales de la salud y los adultos mayores.

En condiciones normales, la investigación y desarrollo de vacunas para uso humano tomarían muchos años y costarían millones de dólares, sobre todo cuando se utilizan nuevas tecnologías que no han sido examinadas de manera exhaustiva. Pero, ya que en el mercado no existían vacunas contra el coronavirus, muchos países han autorizado planes de vacunación de emergencia contra el SARS-CoV-2. Estas vacunas se desarrollaron y evaluaron durante el último año en distintas naciones y corresponden a diferentes plataformas, como vacunas de virus inactivados, de virus vivos atenuados, de vectores virales recombinantes, de ARN mensajero, entre otras tecnologías. La vacuna aplicada a los profesionales de la salud en el Perú (Sinopharm) está elaborada a partir de un virus inactivado y ha sido desarrollada por empresa china Sinopharm China National Biotec Group (CNBG) ${ }^{(7,8)}$. Este producto muestra algunas ventajas en las condiciones requeridas para el almacenamiento y transporte. Algunos tipos de vacunas, como las de plataforma ARN mensajero, son bastante inestables a temperatura ambiente $y$ pueden requerir congeladores que, por lo general, no se encuentran en centros de salud rurales lejanos o que no cuentan con sistemas que aseguren la cadena de frío, por lo que las vacunas no refrigeradas pueden ser una solución más viable para estos lugares ${ }^{(9)}$.

En el Perú, la mayoría de los trabajadores de la salud han sido vacunados; entre ellos, el $84,42 \%$ de los médicos ${ }^{(10)}$. El Ministerio de Salud ha aplicado a su personal dos dosis de la vacuna china Sinopharm. Esta vacuna, de probada inmunogenicidad y seguridad (11,12), enfrenta al sistema inmune de los vacunados con la totalidad de antígenos virales, pues se basa en la inactivación del virus por efecto de la droga B-propionolactona ${ }^{(13)}$.

La capacidad del sistema inmune de limitar la infección viral a las células depende de diversos factores, como la inducción de anticuerpos neutralizantes (14). En consecuencia, es relevante evaluar la capacidad de una vacuna de inducir la formación de estos anticuerpos y determinar los factores que pueden influir en su presencia en el suero de los individuos vacunados. El presente trabajo describe la información de la base de datos del centro Gamma Médica Servicios Médicos de la ciudad de Lima, donde han asistido profesionales sanitarios vacunados para cuantificar los anticuerpos neutralizantes en el suero. En este trabajo solo se ha incluido a las personas que recibieron la vacuna SINOPHARM, y comparó la presencia de anticuerpos neutralizantes en dos grupos: las personas con diagnóstico de COVID-19 antes de la vacunación y los individuos que no tuvieron este diagnóstico.

\section{MATERIALES Y MÉTODOS}

\section{Diseño y población de estudio}

Investigación descriptiva y de diseño transversal. Los datos analizados corresponden a 117 profesionales de la salud vacunados con dos dosis de la vacuna Sinopharm, que acudieron al centro Gamma Médica en los meses de marzo y abril del 2021 para detectar anticuerpos neutralizantes contra SARS-CoV-2 mediante la técnica de inmunoensayo de electroquimioluminiscencia (eCLIA) de Shenzhen Lifotronic Technology Co., Ltd. Se excluyeron ocho registros que no consignaban el tipo de vacuna administrada.

\section{Variables y mediciones}

Se consideraron las variables edad, sexo, antecedentes de infección con SARS-CoV-2 (diagnosticados con COVID-19) y concentración de anticuerpos neutralizantes.

\section{Análisis estadístico}

Los datos se analizaron mediante la estadística descriptiva para las variables en estudio. Para el análisis de anticuerpos neutralizantes se utilizó como punto de corte la detección de $10 \mathrm{UA} / \mathrm{mL}$ de anticuerpos, según la recomendación del fabricante de la técnica de detección utilizada (Shenzhen Lifotronic Technology Co., Ltd.). Las diferencias estadísticas entre los valores de lectura de anticuerpos neutralizantes, entre individuos que tuvieron o no COVID-19, se determinó mediante la prueba $t$ de Student para muestras no emparejadas, con un nivel de confianza de $95 \%$. Los datos se analizaron con el software estadístico IBM SPSS v.27. 


\section{Consideraciones éticas}

Los investigadores garantizan la confidencialidad de los

datos utilizados en este estudio.

\section{RESULTADOS}

En el estudio, 117 profesionales de la salud recibieron dos dosis de la vacuna anti-SARS-CoV-2 desarrollada por la empresa Sinopharm. De ellos, el 52,10 \% son de sexo masculino; el 79,50\% tiene más de 42 años, y el 17,90\% manifestó haber tenido COVID-19 antes de ser vacunados (Tabla 1).

Tabla 1. Características de la población de estudio

\begin{tabular}{|c|c|c|}
\hline & Frecuencia & $\%$ \\
\hline \multicolumn{3}{|l|}{ Sexo } \\
\hline Masculino & 61 & 52,10 \\
\hline Femenino & 56 & 47,90 \\
\hline \multicolumn{3}{|l|}{ Edad (años) } \\
\hline$\leq 30$ & 5 & 4,30 \\
\hline $31-42$ & 19 & 16,20 \\
\hline $43-54$ & 46 & 39,30 \\
\hline $55-65$ & 37 & 31,70 \\
\hline Más de 66 & 10 & 8,50 \\
\hline \multicolumn{3}{|l|}{ COVID-19 previa } \\
\hline Sí & 21 & 17,90 \\
\hline No & 96 & 82,10 \\
\hline
\end{tabular}

En la tabla 2 se muestra el análisis de los datos correspondientes a la presencia de anticuerpos neutralizantes contra SARS-CoV-2 en los individuos vacunados. El valor de corte considerado para definir la presencia o no de anticuerpos neutralizantes en el suero es
$10 \mathrm{UA} / \mathrm{mL}$, mientras que el valor máximo reportado en la base de datos fue de $600 \mathrm{UA} / \mathrm{mL}$. Se observa que el 47,90 $\%$ de los individuos vacunados no desarrollaron anticuerpos neutralizantes contra el nuevo coronavirus.

Tabla 2. Presencia de anticuerpos neutralizantes en individuos con o sin diagnóstico de COVID-19 antes de la vacunación

\begin{tabular}{|lrr|}
\hline \multicolumn{3}{|c}{ Frecuencia } \\
\hline Lectura $(\mathrm{UA} / \mathrm{mL})$, total pacientes & & \\
\hline$\leq 10$ & 55 & 47,90 \\
\hline$>11$ & 62 & 52,10 \\
\hline Lectura $(\mathrm{UA} / \mathrm{mL})$, con COVID-19 previa & & \\
\hline$\leq 10$ & 5 & 23,80 \\
\hline$>11$ & 16 & 76,20 \\
\hline Lectura $(\mathrm{UA} / \mathrm{mL})$, sin COVID 19 previa & & \\
$\leq 10$ & 50 & 52,10 \\
\hline$>11$ & 46 & 47,90 \\
\hline
\end{tabular}

Al comparar la presencia de anticuerpos neutralizantes en el suero de individuos vacunados que habían sido o no diagnosticados con COVID-19, se detectó una mayor frecuencia de individuos con anticuerpos neutralizantes en el grupo con diagnóstico previo de COVID-19 (76,20\% vs. $47,90 \%)$. 
Se comparó el valor de los anticuerpos neutralizantes cuantificados en individuos con o sin diagnóstico previo de COVID-19 y, como se observa en la tabla 3, hay una diferencia estadísticamente significativa a favor de los individuos que tuvieron COVID-19 antes de ser vacunados $(p<0,001)$.

Tabla 3. Concentración de anticuerpos neutralizantes detectados en individuos con o sin diagnóstico de COVID-19 antes de la vacunación

\begin{tabular}{cccrc} 
CoVID-19 previa & Frecuencia & Media de lectura (UA/mL) & IC $95 \%$ & $p$ \\
\hline Sí & 21 & 75,96 & $17,14-134,79$ & $<0,001$ \\
No & 96 & 12,24 & $10,15-14,32$ & \\
\hline
\end{tabular}

\section{DISCUSIÓN}

El rol crucial de los profesionales de la salud en el manejo de la pandemia por SARS-CoV-2 es innegable, y por ello se denomina a este grupo como personal de primera línea. En consecuencia, es sumamente importante garantizar que su trabajo continúe para cumplir, de manera óptima, la invaluable labor de prevención y atención de los enfermos. Las infecciones del personal sanitario contraídas durante el trabajo se han descrito desde el inicio de la pandemia y han provocado intensas discusiones sobre el equipo de protección personal (EPP) adecuado (15), tal como reportan Wu et al., cuyo trabajo muestra una tasa de infección del 3,80\% entre los profesionales de la salud en Wuhan, China ${ }^{(16)}$.

En todo el mundo, la vacunación masiva para inmunizar a la población contra el virus SARS-CoV-2 es una estrategia muy importante para controlar la pandemia y reducir la morbimortalidad asociada. En este sentido, diversas plataformas vacunales han sido la base para el desarrollo de productos contra este virus, y han empleado material genético ( $A R N$ y $A D N$ ), antígenos (proteina $S$ ) y virus inactivados (3). Instituciones como Pfizer, Moderna, AstraZeneca, Gamaleya, Johnson \& Johnson y Sinopharm lideran el escalado productivo de vacunas y su distribución mundial (4).

A la fecha, en el Perú, un poco más de 1,3 millones de personas han recibido al menos dos dosis de alguna de las vacunas contra SARS-CoV-2; y cerca de 3 millones, al menos una dosis ${ }^{(17)}$. En la mayoría de los casos, los profesionales sanitarios han sido inmunizados con el producto de la empresa china Sinopharm ${ }^{(10)}$. La Organización Mundial de la Salud (OMS) reporta que esta vacuna ha demostrado un adecuado perfil de seguridad y una eficacia cercana al $80 \%$, lo que ha impulsado su uso en todo el mundo (12). No obstante, se ha planteado la necesidad de varias dosis de reforzamiento para incrementar su inmunogenicidad a largo plazo ${ }^{(18)}$.

La vacuna de Sinopharm fue desarrollada a partir de una variante viral inactivada con la droga B-propionolactona ${ }^{(13)}$.
Las vacunas que utilizan esta plataforma vacunal inducen la respuesta inmune contra diferentes antígenos virales, Aunque, por lo regular, son necesarias múltiples dosis para generar una respuesta de memoria de larga duración. Estos productos se caracterizan por su seguridad, fácil manipulación y almacenamiento, y por tener bajos costos de producción ${ }^{(4)}$.

La información de la base de datos del Centro Gamma Médica Servicios Médicos incluye los valores de la concentración de anticuerpos neutralizantes en el suero de 117 profesionales sanitarios que recibieron dos dosis de la vacuna Sinopharm. Encontramos un porcentaje mayor de personas con anticuerpos neutralizantes en el grupo que había sido previamente diagnosticado con COVID-19. Así mismo, al comparar la concentración de estos anticuerpos (expresada como $\mathrm{UA} / \mathrm{mL}$ ) en las personas vacunadas que habían padecido o no COVID-19 antes de la vacunación, el valor resulta significativamente más alto en los que habían tenido la enfermedad.

Las vacunas fabricadas a partir de patógenos completos, como la desarrollada por Sinopharm, pueden enfrentar al sistema inmune a todo el mundo antigénico del microorganismo. Es decir que, a diferencia de otras vacunas ${ }^{(19,20)}$, la respuesta inmune que induce no está focalizada en antígenos directamente ligados a la infección, como la proteína S. Haber padecido COVID-19 antes de ser vacunado estimula la actividad del sistema inmune, lo cual es reforzado luego por la propia vacunación. De alguna manera, en los individuos que han tenido COVID-19, una vacuna que incluya todos los antígenos virales se comportaría como dosis de reforzamiento tras la infección natural. El resultado de este estudio es valioso y apunta a la necesidad de incluir una dosis adicional de esta vacuna, sobre todo en el personal de alto riesgo.

Contribuciones de los autores: Arturo Pareja Cruz y Jesús Demóstenes Gonzáles Moscoso: obtención de los datos. Pedro Javier Navarrete Mejía: análisis de los datos, redacción y edición. Joel de León: redacción y edición. Julio César Luque Espino: redacción y edición final. 
Fuentes de financiamiento: El artículo ha sido financiado por los autores.

Conflicto de interés: Los autores declaran no tener ningún conflicto de interés.

\section{REFERENCIAS BIBLIOGRÁFICAS}

1. Samudrala PK, Kumar P, Choudhary K, Thakur N, Wadekar GS, Dayaramani R, et al. Virology, pathogenesis, diagnosis and in-line treatment of COVID-19. Eur J Pharmacol. 2020; 883: 173375.

2. He F, Deng Y, Li W. Coronavirus disease 2019: What we know?. J Med Virol. 2020; 92(7): 719-25.

3. Taylor PC, Adams AC, Hufford MM, de la Torre I, Winthrop K, Gottlieb RL. Neutralizing monoclonal antibodies for treatment of COVID-19. Nat Rev Immunol. 2021; 21(6): 382-93.

4. Forni G, Mantovani A, COVID-19 Commission of Accademia Nazionale dei Lincei, Rome. COVID-19 vaccines: where we stand and challenges ahead. Cell Death Differ. 2021; 28(2): 626-39.

5. Hofman K, Shenoy GN, Chak V, Balu-Iyer SV. Pharmaceutical aspects and clinical evaluation of COVID-19 vaccines. Immunol Invest. 2021; 1 -37.

6. Kyriakidis NC, López-Cortés A, Vásconez Gonzáles E, SARS-CoV-2 vaccines strategies: a comprehensive review of phase 3 candidates. NPJ Vaccines. 2021; 6(1): 28.

7. Awadasseid A, Wu Y, Tanaka Y, Zhang W. Current advances in the development of SARS-CoV-2 vaccines. Int J Biol Sci. 2021; 17(1): 8-19.

8. Padron-Regalado E. Vaccines for SARS-CoV-2: lessons from other coronavirus strains. Infect Dis Ther. 2020; 9(2): 1-20.

9. Izda V, Jeffries MA, Sawalha AH. COVID-19: A review of therapeutic strategies and vaccine candidates. Clin Immunol. 2021; 222: 108634.

10. Colegio Médico del Perú. Vacunómetro-CMP [Internet]. Lima: CMP; 2021. Disponible en: https://www.cmp.org.pe/vacunometro-cmp/

11. Xia S, Zhang $Y$, Wang $Y$, Wang $H$, Yang $Y$, Gao GF, et al. Safety and immunogenicity of an inactivated SARS-CoV-2 vaccine, BBIBP-CorV: a randomised, double-blind, placebo-controlled, phase $1 / 2$ trial. Lancet Infect Dis. 2021; 21(1): 39-51.

12. World Health Organization. The Sinopharm COVID-19 vaccine: what you need to now [Internet]. WHO; 2021. Disponible en: https:// www.who.int/news-room/feature-stories/detail/the-sinopharmcovid-19-vaccine-what-you-need-to-know

13. Wang H, Zhang Y, Huang B, Deng W, Quan Y, Wang W, et al. Development of an Inactivated Vaccine Candidate, BBIBP-CorV, with Potent Protection against SARS-CoV-2. Cell. 2020; 182(3): 713-21.e9.

14. Murin $C D$, Wilson IA, Ward AB. Antibody responses to viral infections: a structural perspective across three different enveloped viruses. Nat Microbiol. 2019; 4(5): 734-47.

15. Sommerstein R, Fux CA, Vuichard-Gysin D, Abbas M, Marschall J, Balmelli $C$, et al. Risk of SARS-CoV-2 transmission by aerosols, the rational use of masks, and protection of healthcare workers from COVID-19. Antimicrob Resist Infect Control. 2020; 9(1): 100.

16. Wu Z, McGoogan JM. Characteristics of and Important Lessons From the Coronavirus Disease 2019 (COVID-19) Outbreak in China: Summary of a Report of 72314 Cases From the Chinese Center for Disease Control and Prevention. JAMA. 2020; 323(13): 1239-42.

17. Ministerio de Salud. Campaña Nacional de Vacunación contra la COVID-19 [Internet]. MINSA: Lima; 2021. Disponible en: https:// www.gob.pe/institucion/pcm/campa\%C3\%B1as/3451-campananacional-de-vacunacion-contra-la-covid-19

18. Mallapaty S. China's COVID vaccines are going global - but questions remain. Nature. 2021; 593(7858): 178-9.
19. Sahin U, Muik A, Derhovanessian E, Vogler I, Kranz LM, Vormehr M, et al. COVID-19 vaccine BNT162b1 elicits human antibody and TH1 T cell responses. Nature. 2020; 586(7830): 594-9.

20. Logunov DY, Dolzhikova IV, Shcheblyakov DV, Tukhvatulin Al, Zubkova OV, Dzharullaeva AS, et al. Safety and efficacy of an rAd26 and rAd5 vector-based heterologous prime-boost COVID-19 vaccine: an interim analysis of a randomised controlled phase 3 trial in Russia. Lancet. 2021; 397(10275): 671-81.

\section{Correspondencia:}

Arturo Pareja Cruz

Dirección: Av. Alameda del Corregidor 1531, Urb.

Los Sirius, La Molina. Lima, Perú.

Teléfono: 3652300

Correo electrónico: aparejac@usmp.pe

Recibido:01 de junio de 2021 Revisado: 04 de junio de 2021 Aprobado: 10 de junio de 2021

(c) La revista. Publicado por Universidad de San Martín de Porres, Perú. (cc) $\mathbf{B r}$ Licencia de Creative Commons Artículo en acceso abierto bajo términos de Licencia Creative Commons Atribución 4.0 Internacional. (http://creativecommons.org/licenses/by/4.0/)

\section{ORCID iDs}

Arturo Pareja Cruz Joel De León Delgado Pedro Javier Navarrete Mejía Julio César Luque Espino
- https://orcid.org/0000-0002-5988-5515 () https: / / orcid.org/0000-0002-3664-8023

(1) https: / / orcid.org/0000-0002-9809-6789

가 https: / / orcid.org/0000-0001-8868-2883 